Journal of Physics and Its Applications

Journal homepage: https://ejournal2.undip.ac.id/index.php/ipa/index

\title{
Comparison of I-V Curves Between the Experiment of Corona Discharge on Gradient Line-To-Plane (GL-P) Configuration and The Mathematical Approach
}

\author{
Susilo Hadi1 , Asep Yoyo Wardaya1, ${ }^{*}$, Zaenul Muhlisin ${ }^{1,2}$, Jatmiko Endro Suseno1, Pandji Triadyaksa ${ }^{1,2}$, \\ Ali Khumeni ${ }^{1}$, and Muhammad Nur ${ }^{1,2}$ \\ ${ }^{1}$ Department of Physics, Faculty of Science and Mathematics, Diponegoro University, Semarang, Indonesia \\ ${ }^{2}$ Center for Plasma Research, Diponegoro University, Semarang, Indonesia \\ ${ }^{*}$ Corresponding author:asepyoyowardayafisika@gmail.com
}

\section{A RTICLE IN F O}

\section{Article history:}

Received: 1 March 2021

Accepted: 3 May 2021

Available online: 28 May 2021

\section{Keywords:}

The value of multiplication factor $k$

Corona discharge

Geometrical approach

GL-P electrode configuration

I-V characteristic curve

\begin{abstract}
A B S T R A C T
Research has been conducted on the comparison of the I-V characteristic curve between mathematical study and experiment of the generation of negative DC corona discharge in the Gradient Line-to-Plane (GL-P) electrode configuration. The reason for this research is to calculate the suitability of the corona discharge electrical current between the mathematical and experimental study. The active electrode used has length $2 \mathrm{~cm}$ and height $4 \mathrm{~cm}$. This research is conducted with variations in the sharpness angle of the active electrode $(\theta) 30^{\circ}, 45^{\circ}$, and $60^{\circ}$ and variations in the distance between the electrodes $(D) 2 \mathrm{~cm}, 3 \mathrm{~cm}$, and $4 \mathrm{~cm}$. The mathematical formulation of the value of the corona discharge electrical current in the configuration of the GL-P electrode is obtained by using the geometric concept approach, which is the formulation of the capacitance value of the ordinary electrical circuit, with the addition of the multiplication factor value $\mathrm{k}$ in the sharp area of the active electrode, because in that area the greatest plasma flow distance is obtained. The value of the multiplication factor $k$ is obtained by fitting the curve between mathematical study and experiments. The I-V curve between the mathematical study and the corona discharge generation experiment has a high degree of similarity with the smallest percentage contacting point of $37.50 \%$.The value of the multiplication factor $k$ is influenced by the sharpness angle of the active electrode shape and the distance between the electrodes.
\end{abstract}

\section{Introduction}

Plasma was first defined by Irving Langmuir as ionized gas in an electrical discharge in 1928. The name Plasma inspired by blood plasma is described as a region that shows the balance of charges between ions and electrons in an electric discharge [1]. Thus, Plasma is known as the quasi-neutral state between the gas charge and the gas particles themselves which shows the collective nature of plasma [2]. One kind of plasma discharges produced with free air medium is corona discharge [3]. The corona discharge is formed by providing an asymmetrical two-electrode configuration as in the point-plane electrode configuration [4]. This model has an arrangement of a pair of electrodes including one electrode with a sharp point as a point electrode and the other electrode in the shape of a horizontal rectangle.

The corona discharge process begins with a small and flat variation in the value of the electric current at the start of the voltage. This is called the Townsend discharge. After that, there is a sharp increase in the value of the electric current as a function of voltage so that a Spark discharge is occurred and even become an Arc discharge. Corona discharge occurs between Townsend discharge and the transition area to Independent Discharge [5]. This process can be represented in the form of a current-voltage (I-V) characteristic curve on corona discharge. The value of the electric current obtained on the I-V characteristic curve can be formulated by considering the circumstances that occur in the corona discharge. The value of the electric current generated in the corona discharge is very large compared to the value of the electric current in an ordinary electric circuit [6]. This is due to various circumstances in the corona discharge such as electrohydrodynamic (EHD) [7], ionic wind phenomena [8], electrostatic precipitation [9], convective heat transfer [10], and so on. The number of circumstances occurring in the corona discharge makes it difficult to formulate the value of electric current as a function of voltage in order to obtain the precise value of the electric current. Therefore, it is 
necessary to make a specific approachthrough modelling in the mathematical formulation of the value of the electric current in the corona discharge.

There is a new approach used in the mathematical formulation of the value of electric current. This approach models the formulation of the value of electric current by adopting the capacitance value of the ordinary electric circuit in the Line-toPlane electrode configuration [6]. The value of the electric current is obtained by modified formulation of the capacitance, which is the addition of the multiplication factor $k$ on the sharp of electrode curvature. This value shows the characteristic of the appearance of an enormous corona discharge electric current on a sharp electrode geometric curvature because the area has a large potential gradient. The value of the multiplication factor $k$ cannot be calculated mathematically but can be obtained by fitting the curve to the experimental result. A large value of the multiplication factor $k$ indicates that the value of the electric current generated by the corona discharge is extremely greater than by an ordinary electric circuit. The sharper shape of the electrode geometric curvature used, the greater the value of the resulting corona discharge current will be [6]. The approach used in the formulation has no further validation in the term of its application to other electrode configurations. The degree of appropriateness of curve fitting utilized has no using a statistical approach in order to know the quantity of curve fit precision. Moreover, the study of the effect of different sharpness angle on the curvature of the electrode geometry toward the determination of the value of the multiplication factor $k$ has not been carried out.

Therefore, this research is conducted to recognize further the mathematical formulation modelling approach of the value of the corona discharge electric current applied by [6] which can be used for different electrode configurations. Moreover, this study determines the level of similarity of the I-V characteristic curve between mathematical study and experimental result of corona discharge in free air using a statistical approach and analyze the effect of determining the value of the multiplication factor $k$ toward the sharpness angle and the distance between the electrodes. The electrode configuration used in this study is the Gradient of Line-to-Plane (GL-P) electrode configuration. This research is conducted using an active electrode containing $2 \mathrm{~cm}$ length and $4 \mathrm{~cm}$ height and there are 2 kinds of variations, which are variations in the sharpness of the active electrode and the distance between the electrodes.

\section{Mathematical Method Approximation}

The mathematical model formulation is calculated by using the approach of the electrode geometry, which is the formulation of capacitance in the Gradient Line-to-Plane (GL-P) electrode configuration shown in Fig. 1a). The formula is the value of the corona discharge electric current as a function of the voltage in the GL-P electrode configuration obtained from the advanced formulation of the electric charge and electric field function [6] as shown in equation 1.

$$
I=-\frac{\mu_{0}\left(V-V_{i}\right)^{2}\left(C_{t o t}\right)^{3}}{\varepsilon_{0}^{2}\left(\delta a+\delta^{2}\right)^{2}}
$$

Where $I$ is the electric current, $V$ is the voltage, $C_{\text {tot }}$ is the total capacitance value, $a$ is the length of the active electrode, and $\delta$ is the thickness of the active electrode.

The mathematical formulation begins by calculating the capacitance equation in the rectangular-plane electrode configuration [6], as shown in Fig. 1b). The configuration is adopted to determine the capacitance of the GL-P electrode configuration. Both configurations consist a very thin active electrode standing perpendicularly to the horizontal passive electrode. The capacitance value is generated in equation 2 where $b$ is the plate height and $D$ is the distance between two electrodes.

$$
C=\varepsilon_{0} a \ln \left(\frac{b}{D}+1\right)
$$

Thus, the active electrode element is divided into a quadrangle and a right-triangle. The equation of capacitance in the rectangular-plane configuration is used to determine the capacitance value for the quadrangular and right-triangular elements. In the right-triangular element, the capacitance value is given the multiplication factor value $k$ on the sharpness of electrode geometric curvature. The total capacitance value is obtained by adding up the capacitance values on the quadrangular and righttriangular elements. By the total capacitance value, the equation of the electric current of the corona discharge generation in the GL-P electrode configuration is generated.

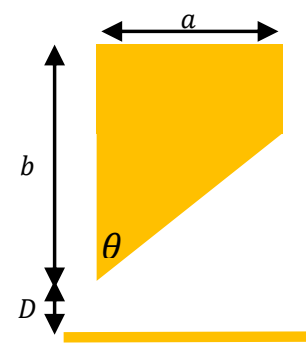

(a)

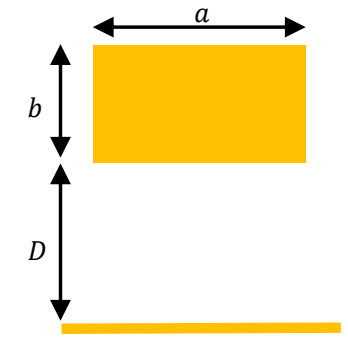

(b)

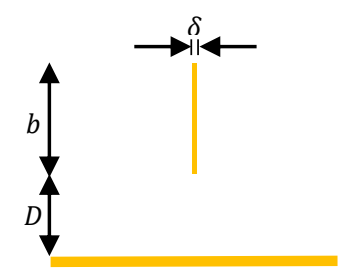

(c)

Fig 1:a) GL-P Electrode Configuration,b) Rectangularto-Plane Electrode Configuration, and c)Side View of Configurations. 


\section{Capacitance Calculation}

The capacitance value of the Gradient Line-to-Plane (GL-P) electrode configuration is calculated by dividing the GL-P electrode configuration into 2 plates, which are a quadrangular plate and a righttriangular plate as shown in Fig. 2. The capacitance value of each element of The GL-P electrode configuration can be written in equation 3 .

$$
d C_{G L-P}=\varepsilon_{0} d u \ln \left(\frac{w}{h}+1\right)
$$

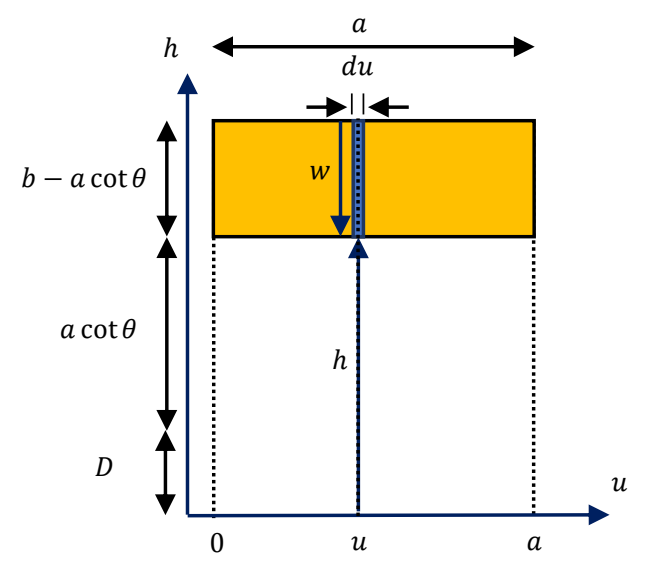

a)

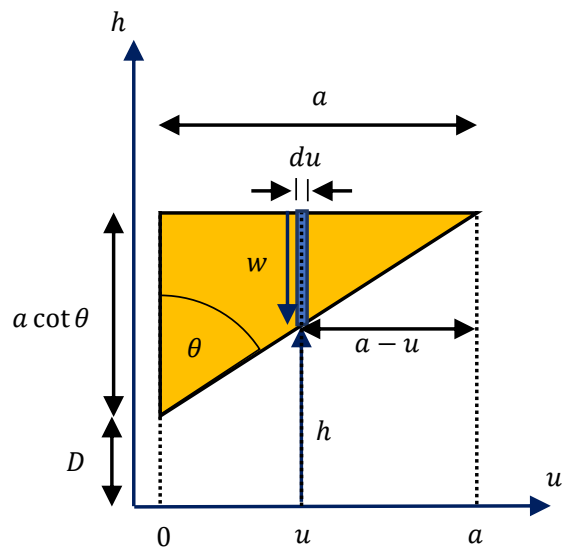

b)

Fig 2: Elements of the GL-P Electrode Configuration a) quadrangular plate element andb) right-triangular element.

The first plate element is a quadrangular plate. Based on Fig. 2a), the values of $w$ and $h$ are obtained. The total capacitance value of the quadrangular plate is generated by adding the capacitance through the integration of each element over the distance $u$ from 0 to $a$. By adding the values of $w$ and $h$ in equation 3, the total capacitance value of the rectangular plate is obtained in equation 4 .

$$
\begin{aligned}
C_{\text {quadrangle }}=\varepsilon_{0} & \ln \left(\frac{b+D}{a \cot \theta+D}\right) \int_{u=0}^{a} d u \\
= & \varepsilon_{0}\{a \ln (b+D) \\
& -a \ln (a \cot \theta+D)\}
\end{aligned}
$$

The second plate element is a right-triangular plate. Based on Fig. 2b), the values of $w$ and $h$ are obtained. The total capacitance value of the right- triangular plate is calculated by integrating each element over the distance of $u$ from 0 to $a$. By substituting the values of $w$ and $h$ into equation 3 , the total capacitance value of the right-triangular plate can be written in equation 5 .

$$
C_{\text {triangle }}=\varepsilon_{0} \int_{u=0}^{a} d u \ln \left(\frac{a \cot \theta+D}{a \cot \theta+D-w}\right)
$$

It is necessary to change the variable of $w$ into the variable of $u$ using the ratio between $w$ and $u$ as shown in Fig. 2b). From this comparison, the total capacitance value of the right-triangular plate is obtained as shown in equation 6.

$$
\begin{aligned}
C_{\text {triangle }}=\varepsilon_{0}\{a & \ln (a \cot \theta+D) \\
& \left.-\int_{u=0}^{a} d u \ln (u \cot \theta+D)\right\}
\end{aligned}
$$

To solve this equation, it is required to simplify it by changing the variable of $u$ into another variable, for example, variable $z$. Thus, the total capacitance value of the right-triangular plate is obtained as shown in equation 7 with the integration value limit over the distance of $z$ from $D$ to $a \cot \theta+D$.

$$
\begin{aligned}
C_{\text {triangle }}=\varepsilon_{0}\{a & \ln (a \cot \theta+D) \\
& +\frac{1}{\cot \theta}(a \cot \theta+D)(1 \\
& -\ln (a \cot \theta+D)) \\
& \left.+\frac{1}{\cot \theta} D(\ln (D)-1)\right\}
\end{aligned}
$$

It should be noted that in the formulation of the capacitance value, the value of the multiplication factor $k$ is given on the sharp curvature of active electrode geometric to distinguish the value of the electric current in the corona discharge from the value of the electric current in the ordinary electric circuit. In the case of a right-triangular plate, there is a sharpness of geometric curvature of the active electrode to the passive electrode when the value of $u$ is equal to 0 . This position represents a very high potential gradient value and the appearance of an enormous plasma flow. The multiplication factor $k$ is inserted at $u=0$ or $z=D$ as shown in equation 8 which becomes the total capacitance value on the right-triangular plate.

$$
\begin{aligned}
C_{\text {triangle }}=\varepsilon_{0}\{a & \ln (a \cot \theta+D) \\
& +\frac{1}{\cot \theta}(a \cot \theta+D)(1 \\
& -\ln (a \cot \theta+D)) \\
& +\lim _{k \rightarrow \text { value }} k D \frac{1}{\cot \theta}(\ln (D) \\
& -1)\}
\end{aligned}
$$

The total capacitance value of the GL-P electrode configuration is obtained by adding the total capacitance value of each plate element in this configuration, which is the capacitance value of quadrangular plate is added to the capacitance value of the right-triangular plate so that the equation 9 is generated. 


$$
\begin{aligned}
C_{G L-P}=\varepsilon_{0}\{a \ln ( & b+D) \\
& +\frac{1}{\cot \theta}(a \cot \theta+D)(1 \\
& -\ln (a \cot \theta+D)) \\
& +\lim _{k \rightarrow \text { value }} k D \frac{1}{\cot \theta}(\ln (D) \\
& -1)\}
\end{aligned}
$$

\section{Current Calculation}

While determining the total current value of the corona discharge in the Gradient Line-to-Plane (GL$\mathrm{P})$ electrode configuration, it is necessary to formulate the electric charge and electric field in the corona discharge. If the two electrodes in the GL-P electrode configuration are given a voltage difference of $\Delta V$, where $\Delta V=V-V_{i}$ and $V_{i}$ is the threshold voltage of the corona discharge circuit, then the electric charge value is generated by multiplying the voltage difference with the capacitance value of the GL-P electrode configuration, as shown in equation 10 , and the value of the electric field is using Gauss's law, as shown in equation 11.

$$
\begin{gathered}
q_{G L-P}=\Delta V C_{G L-P} \\
E_{G L-P}=\frac{\Delta V C_{G L-P}}{\varepsilon_{0}\left(\delta a+\delta^{2}\right)}
\end{gathered}
$$

The area of the Gauss surface in the GL-P electrode configuration is defined as $A=\delta a+\delta^{2}$, where $\delta$ is the thickness of the active electrode which is very thin, $\delta \cong 0.1 \mathrm{~mm}$. The surface areas are the regions on the active electrode curvature toward the passive electrode, which are $\delta a$ and another region at the end of the active electrode sharpness curve, $\delta^{2}$. In the GL-P electrode configuration, the sharpness curvature of the active electrodes is only 1. Thus, the value of the electric current is generated as shown in equation 12 by substituting the total capacitance value of the GL-P electrode configuration into equation 1.

\section{Statistical Approach}

There is a statistical approach method used, which is finding the percentage point of contact between mathematical study and experiment.

The percentage point of contact of the value of two electric current data group will show an accurate level of suitability between mathematical study and experiment. While determining the point of contact, it is necessary to extend the value of the electric current in the experiment by widening the value with the standard deviation. Because the I-V curve generated in the experiment shows the distribution of data on second order polynomial, the standard deviation value in the experiment should be a second order polynomial. The value of the standard deviation of the second order polynomial for $\mathrm{N}$ data is as written in equation 13 [11].

$$
\sigma_{I}=\sqrt{\frac{\sum\left(y-A-B V_{i}-C V_{i}^{2}\right)^{2}}{(N-2)}}
$$

With each of the values of $A, B$, and $C$ are obtained through equation 14 .

$$
\left(\begin{array}{ccc}
N & \sum V_{i} & \sum V_{i}^{2} \\
\sum V_{i} & \sum V_{i}^{2} & \sum V_{i}^{3} \\
\sum V_{i}{ }^{2} & \sum V_{i}{ }^{3} & \sum V_{i}^{4}
\end{array}\right)\left(\begin{array}{l}
A \\
B \\
C
\end{array}\right)=\left(\begin{array}{c}
\sum I_{i} \\
\sum V_{i} I_{i} \\
\sum V_{i}{ }^{2} I_{i}
\end{array}\right)
$$

If the percentage of the contact point of the electric current value between the mathematical study and the experimental data gets higher, the level of suitability of the I-V curve will be more accurate.

The multiplication factor $k$ value can be determined after the two data groups show the most accurate level of suitability.

\section{Experimental Method \\ Geometrical Problems}

The experimental design of the negative DC corona discharge experiment in the Gradient of Line-toPlane (GL-P) electrode configuration consists of 2 electrode plates with mutually perpendicular positions as shown in Fig. 3. Both electrode plates are made of copper with a purity of $99.99 \%$. The active electrode is in the form of a right trapezoidal configuration in an upright position which has the length of the plate of $a$, the high side of the plate of $b$, and the very thin of thickness $(\delta \cong 0.1 \mathrm{~mm})$. The active electrode has a shape sharpness angle $(\theta)$ to the vertical axis of the electrode. The passive electrode is in the form of a rectangle (Plane Configuration) which is located under the active electrode with a distance between them of $D$. The passive electrode has a very large area to accommodate all the flow of electric flux that moves from the active electrode to the passive electrode.

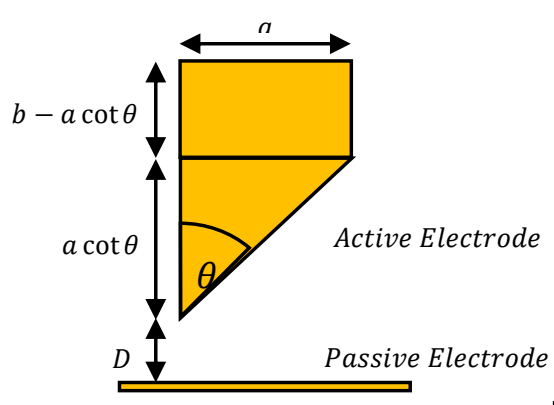

a)

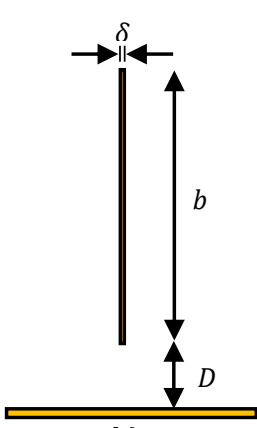

b)
Fig. 3:a)Cross-section of the Gradient of Line-to-Plane (GL-P)Electrode Configuration and b)Side View.

$I_{G L-P}=-\frac{\mu_{0} \varepsilon_{0}\left(a \ln (b+D)+\frac{1}{\cot \theta}(a \cot \theta+D)(1-\ln (a \cot \theta+D))+\lim _{k \rightarrow \text { value }} k D \frac{1}{\cot \theta}(\ln (D)-1)\right)^{3}}{\left(\delta a+\delta^{2}\right)^{2}}\left(V-V_{i}\right)^{2}$ 
The variation of this study is adjusted to experimental data on negative DC corona discharge with GL-P electrode configuration. There are 2 kinds of variations carried out in this study. The first type of variations is the sharpness angle $(\theta)$ to the horizontal side of the active electrode. There are 3 variations of the sharpness angle $(\theta)$, which are $30^{\circ}, 45^{\circ}$, and $60^{\circ}$. The second type of variation is the distance between the electrodes $(D)$. There are 3 variations of the distance between the electrodes $(D)$, which are $2 \mathrm{~cm}, 3 \mathrm{~cm}$, and $4 \mathrm{~cm}$.

\section{Experiment Setup}

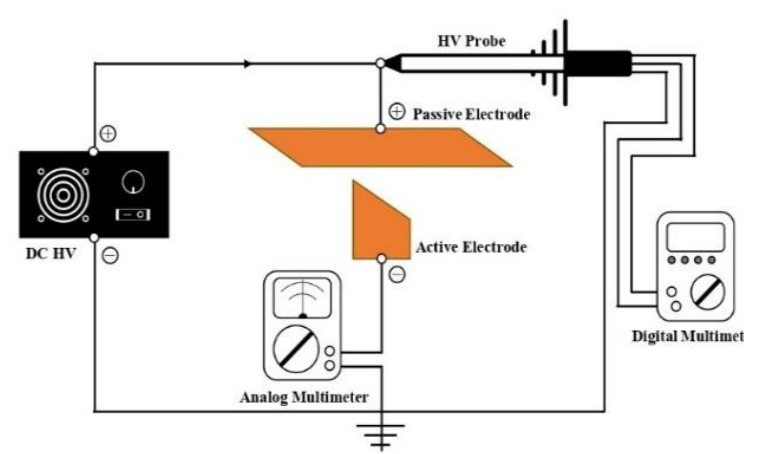

a)

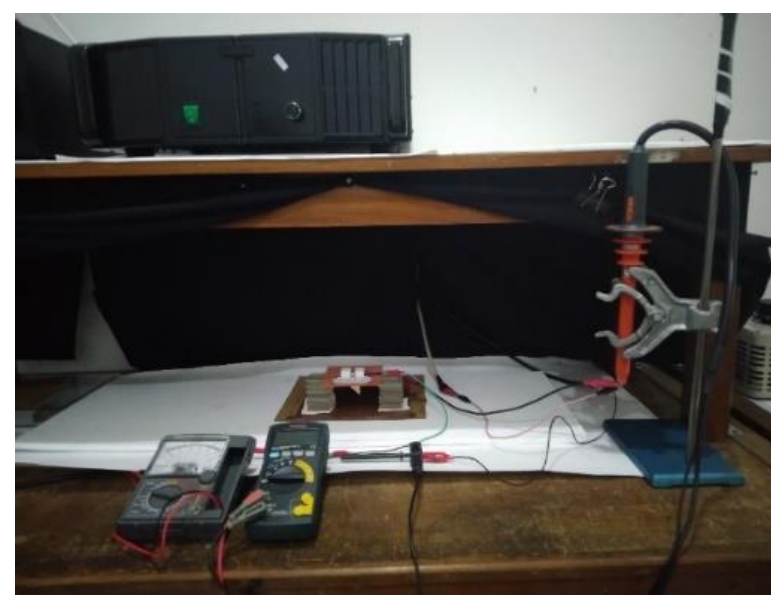

b)

Fig. 4:a) Schematic of Experiment and b) Design of Experiment on the negative DC corona dischargewith GL-P electrode configuration.

The experimental setup is shown in Fig. 4. The experimental design is given a high voltage DC input which has a frequency of $25 \mathrm{kHz}$. The active electrode is connected to the negative pole of the input voltage. In the experimental design, there are tools for measuring the electric current and voltage in the corona discharge circuit. The measurement of the electric current is carried out by using the SANWA analogue multimeter YX-360TREB with a voltage of $220 \mathrm{~V}$ and a frequency of $50 / 60 \mathrm{~Hz}$. The measurement of the potential difference is carried out by using a SANWA digital multimeter type CD771. The circuit is equipped with a High Voltage
Probe with a maximum voltage of DC $40 \mathrm{kV}, \mathrm{AC}$ $28 \mathrm{kV}$, model number PD-28, and serial number 01605733. This probe is applied to convert the value of the potential difference from $\mathrm{kV}$ to $\mathrm{V}$. The dimensions of the active electrode used are the length plate of $2 \mathrm{~cm}$ and the height plate of $4 \mathrm{~cm}$. Corona discharge experiment on the GL-P electrode configuration is carried out in the value range from $0 \mathrm{~V}$ to spark discharge appeared.

\section{Resultsand Discussion Current Analysis}

The electric current value of the negative DC corona discharge generation in the Gradient Line-to-Plane (GL-P) electrode configuration using the electrode geometric shape modelling approach is obtained in equation 12 . In this value, there is a capacitance equation for the GL-P electrode configuration shown in equation 9.

The first term shows the capacitance value of the integration result in the obtuse area of the active electrode. The second term shows the capacitance value of the integration result in the area between the obtuse point and the sharp point of the active electrode or the oblique area of the active electrode. The third term shows the capacitance value of the integration result at the sharp point of the active electrode. In the last term, the added value of the multiplication factor $k$ is given by fitting the current-voltage (I-V) characteristic curve with the negative DC corona discharge experiment with the GL-P electrode configuration. This value indicates an enormous plasma flow at the sharp point of the active electrode because in that area the potential gradient value is very huge so that there is a nonuniform distribution of the electric field in that area. This value will provide a larger value of electric current compared to the value of electric current in an ordinary electric circuit. To obtain a very large current value, the value of the multiplication factor $k$ must be positive and more than one.

The capacitance value resulted will be negative. This is because the value inside of the natural logarithm is in the range from 0 to 1 so that the value of the natural logarithm is negative.

The value of the electric current obtained is directly proportional to the square of the potential difference in the corona discharge circuit with the GL-P electrode configuration. This state shows a similarity to the Townsend relation concerning the corona discharge. The second-order polynomial value at the circuit potential difference illustrates the corona discharge process at the GL-P electrode configuration starting with a small variation in the value of the electric current then increasing a sharp quadratic based on the given potential difference function.

The capacitance value is negative while raised to a power of three and if it is multiplied by the negative sign in the equation, the final value of electric current becomes positive. 


\section{Glow Characteristics}

In this research, the active electrode is used with a length of $2 \mathrm{~cm}$ and a height of $3 \mathrm{~cm}$ including the sharpness angles of $30^{\circ}, 45^{\circ}$, and $60^{\circ}$. The shape of the corona discharge resulted can be seen in Fig. 5 . The discharge produced in Fig. 5 is at a potential difference interval of the circuit between $34 \mathrm{kV}$ to $35 \mathrm{kV}$ and the distance between the electrodes (D)of $2 \mathrm{~cm}$.

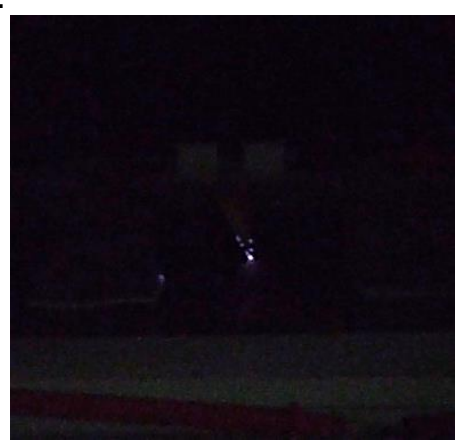

a)

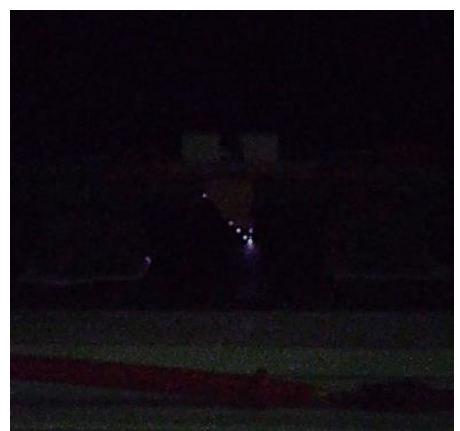

b)

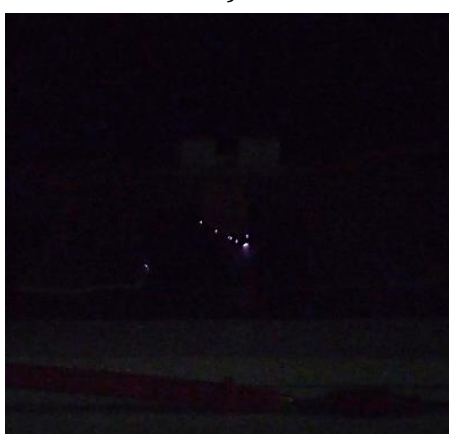

c)

Fig. 5: Corona Discharge of GL-P electrode configuration with the sharpness angle of a) $30^{\circ}$, b) $45^{\circ}$, and c) $60^{\circ}$.

Based on Fig. 5, a discharge of plasma appears in the sharp area of the active electrode. It is because, in the area, the value of the potential gradient is bigger than the surface of the oblique line and the obtuse region of the active electrode. The large value of the potential gradient causes the distribution of the electric field in the sharp area to be not uniform and the corona discharge occurs. On the active electrode with the sharpness angle of $60^{\circ}$, it can be seen that the corona discharge appears in the sharp area, the oblique area, and the obtuse area of the active electrode. The glow resulted in the sharp area is more obvious than in the oblique and obtuse areas. At the sharpness angle of $45^{\circ}$, it can be observed that the corona discharge more clearly appears in the sharp area, but less in some oblique areas and the obtuse area of the active electrode. The glow produced in sharp areas is more apparent than in other areas. Moreover, the glow of the sharp area is more obvious than at the sharpness angle of $60^{\circ}$ and the glow of the obtuse area appears dimmer than at $60^{\circ}$. At the sharpness angle of $30^{\circ}$, it can be perceived that the corona discharge appears only dan most obvious around the sharp area of the active electrode. Furthermore, no glow appears on the obtuse area of the active electrode. Overall, the shape of the corona discharge that occurs in the sharp area is in the form of a crown-shaped glow that spreads to the passive electrode, while the glow that appears in the obtuse area can be ignored because it does not have a significant effect.

Based on the appearance of the corona discharge from different variations in the sharpness angle of the active electrode, an illustration of the distribution of plasma flow can be carried out as shown in Fig. 6.

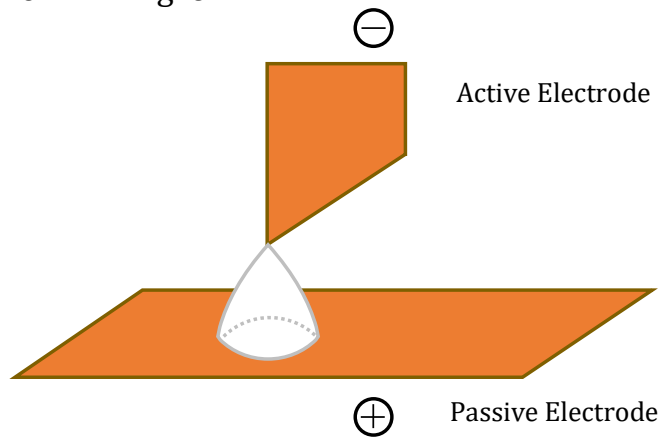

Fig. 6: Illustration of plasma flow distribution on the GL-P Electrode Configuration.

In this figure, it can be noticed that in the sharp area of the active electrode appears a corona discharge with a plasma flow distribution which is shaped cone like a crown.

If the sharpness angle of the active electrode is smaller, the glow produced in the sharp area will be more obvious. This is because the surface of the active electrode becomes sharper and the potential gradient value is very high so that the process of the appearance of the corona discharge will be even larger. This process makes the electronic avalanche and recombination events increase so that more light emission is produced.

It is required to carry out additional analysis concerning the effect of the size shape on the active electrode on the determination of the value of the multiplication factor $k$. Because in the GL-P electrode configuration, there is an obtuse angle on the active electrode, further research is needed whether the obtuse angle has a high enough electric field flux as occurs in the sharp shape of the active electrode. It is also essential to analyze the electric field distribution of the corona discharge on GL-P 
electrode configuration as a piece of validation.

\section{I-V Curve Comparation}

The I-V characteristic curve of the corona discharge between the mathematical study and the experiment is shown in Fig. 7. Overall, it can be seen evidence in the mathematical study. that each I-V characteristic curve of the mathematical study has a high degree of similarity to the experimental curve. The suitability is obtained after curve fitting by determining the value of the multiplication factor $k$.
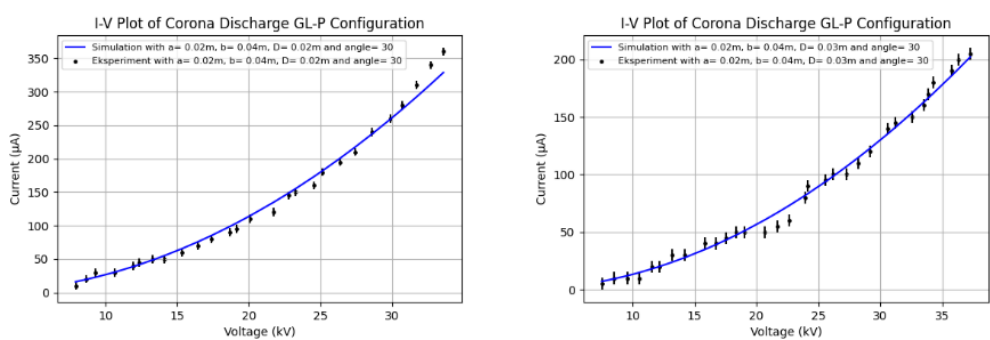

a)
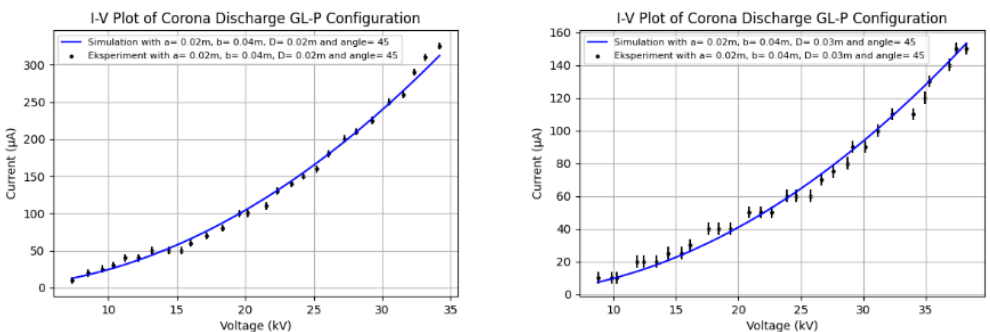

b)

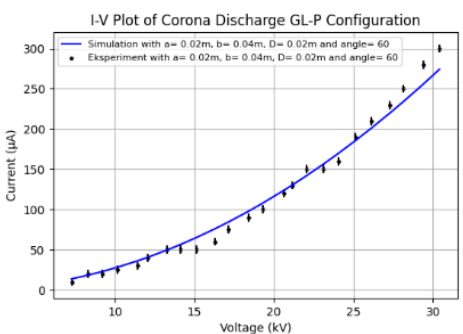

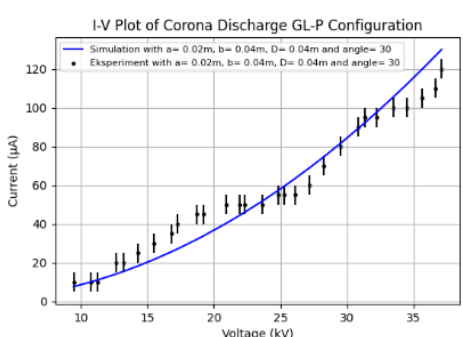
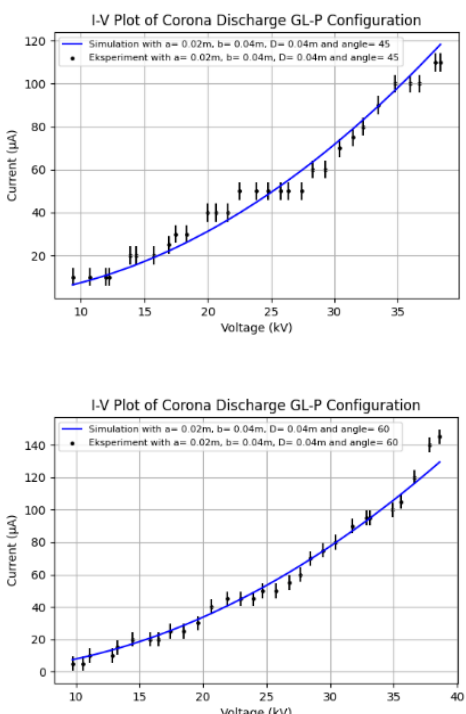

c)

Fig. 7: I-V curves with $V_{i}=0,585 \mathrm{kV}$ sequentially $D=2 \mathrm{~cm}, D=3 \mathrm{~cm}$, and $D=4 \mathrm{~cm}$ on the sharpness angles a) $\theta=$ $30^{\circ}$, b) $\theta=45^{\circ}$, andc) $\theta=60^{\circ}$.

Table 1: the values of the multiplication factor $k$ for each variation of the experiment.

\begin{tabular}{|c|c|c|c|c|c|c|}
\hline$a(m)$ & $\boldsymbol{b}(\boldsymbol{m})$ & $\boldsymbol{\theta}^{0}$ & $D(\boldsymbol{m})$ & $\boldsymbol{k}$ & $S D(\mu A)$ & $\begin{array}{c}\text { Percentage of } \\
\text { Contact Point } \\
(\%)\end{array}$ \\
\hline \multirow{3}{*}{0,02} & \multirow{3}{*}{0,04} & \multirow{3}{*}{30} & 0.02 & 844.5 & 5.9550 & 40.74 \\
\hline & & & 0.03 & 486.9 & 5.1626 & 65.62 \\
\hline & & & 0.04 & 338.2 & 4.9990 & 41.38 \\
\hline \multirow{3}{*}{0,02} & \multirow{3}{*}{0,04} & \multirow{3}{*}{45} & 0.02 & 474.3 & 4.9523 & 53.57 \\
\hline & & & 0.03 & 252.5 & 3.7834 & 58.06 \\
\hline & & & 0.04 & 185.4 & 4.1858 & 56.67 \\
\hline \multirow{3}{*}{0,02} & \multirow{3}{*}{0,04} & \multirow{3}{*}{60} & 0.02 & 284.3 & 5.0660 & 37.50 \\
\hline & & & 0.03 & 164.8 & 4.9795 & 44.83 \\
\hline & & & 0.04 & 110.2 & 4.5005 & 73.33 \\
\hline
\end{tabular}

All curves could be grouped according to each sharpness angle $(\theta)$ as shown in Fig. 8. It can be analyzed the relationship between the distance between the electrodes and the electric current. In the experimental part, it can be seen that the smaller the distance between the electrodes, the resulted I-V curve will be more increase and the discharge is faster in reaching the arc conditions. At 
the sharpness angle of $45^{\circ}$, there is a big range between the I-V curve when $D=0.02 \mathrm{~m}$ and the I-V curve when $D=0.03 \mathrm{~m}$. This condition is different from the curves at both the sharpness angle of $30^{\circ}$ and $60^{\circ}$ which are the distance between the curves for each $D$ value given is almost the same. Moreover, the simulation part shows the same pattern given by the experimental curve so that the simulation results are following the experiment in terms of the effect of the distance between the electrodes on the generated electric current.

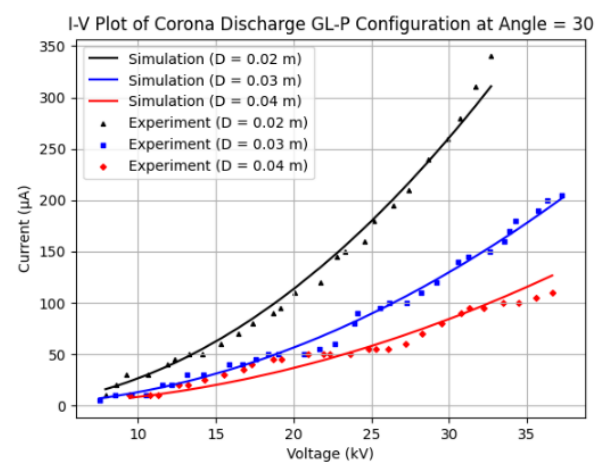

a)

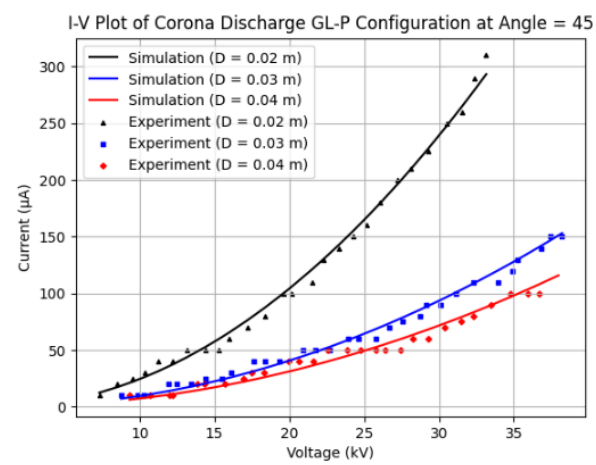

b)

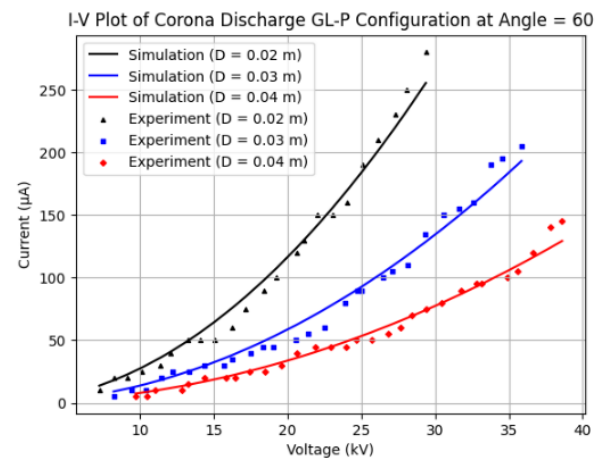

c)

Fig. 8: The $I-V$ curves of Corona Discharge GL-P Configuration ata) $\theta=30^{\circ}$, b) $\theta=45^{\circ}$, and c) $\theta=60^{\circ}$.

In Addition, based on each distance between the electrodes $(D)$, the angle of sharpness of the active electrode can be analyzed for its effect on the electric current as shown in Fig. 9. The experimental curves shown by the three curves indicate that when the sharpness angle of $45^{\circ}$, the I-V curve is more decrease than the others and the highest I-V curve is generally obtained at the sharpness angle of $30^{\circ}$. The same thing is shown in the simulation curves which make them consistent with the experimental results in terms of the effect of the sharpness angle of the active electrode on the resulted electric current.

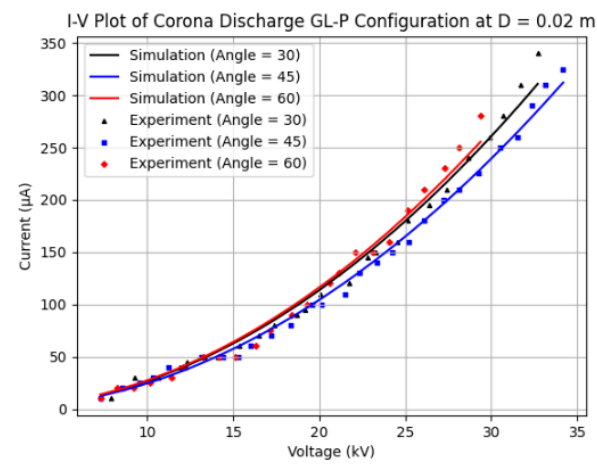

a)

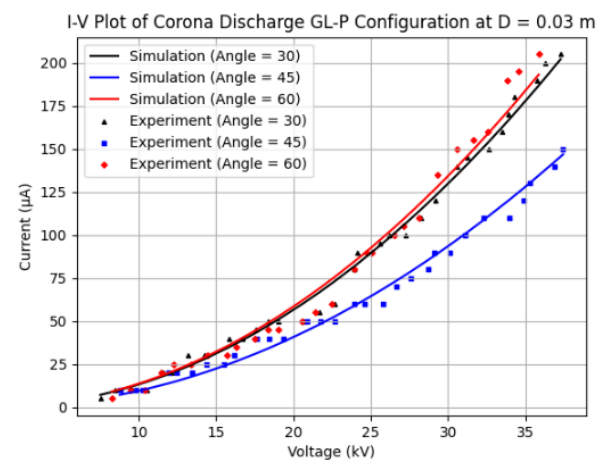

b)

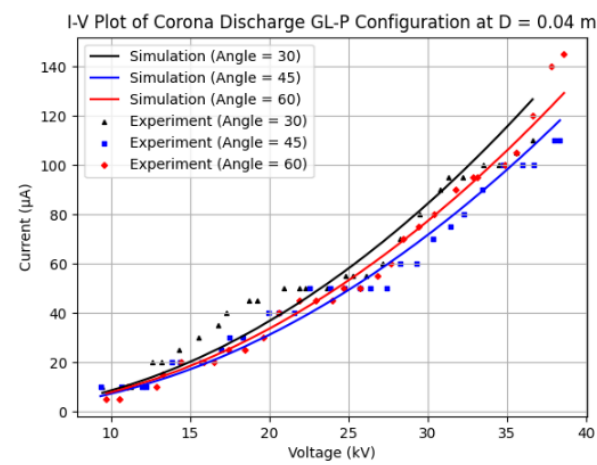

c)

Fig. 9: The $I-V$ curves of Corona Discharge GL-P Configuration at a) $D=0.02 \mathrm{~m}$, b) $D=0.03 \mathrm{~m}$, and c) $D=0.04 \mathrm{~m}$.

\section{Multiplication Factork}

Based on the data, the relationship between the values of the multiplication factor $k$ and the sharpness angle of the active electrode is shown in Fig. 10.

Based on this figure, it can be seen that when the variation in the sharpness angle of the active electrode is greater, the multiplication factor value obtained will be smaller. This circumstance shows that the sharper the active electrode geometric curvature, the greater the value of multiplication factor $k$ obtained. The sharpness of the active electrode geometric curvature exhibits a high 
potential gradient value resulting in an increase in the flux of the electric field and the appearance of a very large plasma current in the area.

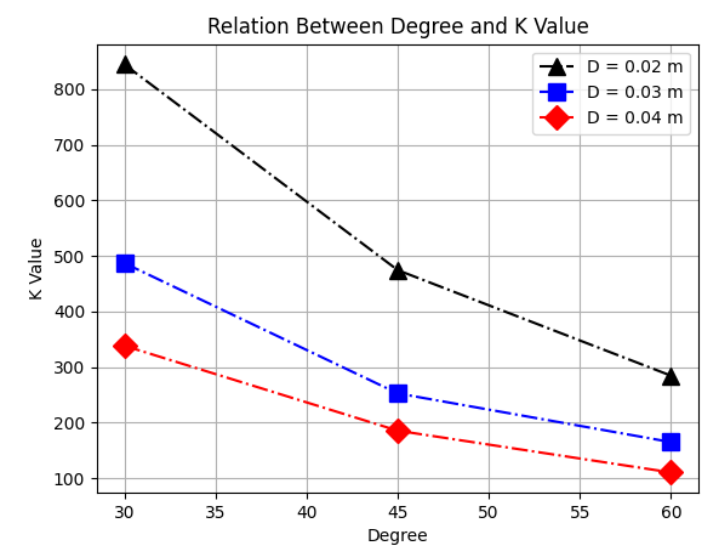

Fig. 10: The relationship between the value of the multiplication factor $k$ and the Sharpness Angle of the Active Electrode.

Furthermore, when the variation of the distance between the electrodes from each sharpness angle of the shape is greater, the value of the multiplication factor $k$ will be getting smaller. This condition happens because the capacitance value in the GL-P electrode configuration is influenced by the distance between the electrodes. The capacitance value is inversely proportional to the distance between the electrodes on a parallel plate. When the distance between the electrodes in the corona discharge generation is enlarged, the capacitance value will be smaller, as a result, the value of the multiplication factor $k$ obtained will be smaller. This condition occurs because the process of the appearance of an electric current in the GL-P electrode configuration requires a higher voltage, especially in the sharp geometric curvature of the active electrode.

This research is conducted to develop a method to determine the electric current in the generation of corona discharge with various types of electrode configurations. The method adopted is an approach to fundamental physics calculations on ordinary electrical circuit[6], namely the capacitance of the electrode circuit. In this method, it is necessary to carry out further analysis regarding the multiplication factor $\mathrm{k}$ obtained because the corona discharge event occurs due to physical phenomena such as electrohydrodynamic[7], ionic wind phenomenon[8], and so on. Research on the effect of size shapes also requires to be done to observe its effect on determining the value of $\mathrm{k}$. In addition, further analysis of the discharge spread visualization and simulation is also important for the $k$ value. There are several great purposes that this research can become a reference in determining the corona discharge electric current, describing the shape of the I-V curve configuration used and the utilization of industrial aspects.

\section{Conclusion}

The mathematical formulation of the value of the electric current for the generation of corona discharge in the Gradient of Line-to-Plane (GL-P) electrode configuration is obtained using the geometrical curvature concept approach of the electrode used, which is the formulation of the capacitance value of the ordinary electric circuit with the addition of the value of multiplication factor $k$ in the sharp area of the active electrode. The greatest plasma flow distribution occurs in the sharp area of the active electrode in the GL-P electrode configuration. The I-V curve between the mathematical study and the corona discharge generation experiment has a great degree of similarity with the smallest percentage point of contact $37.50 \%$. The value of the multiplication factor $k$ is obtained by fitting the curve between mathematical study and experiment. This value is influenced by the sharpness angle of the active electrode shape and the distance between the electrodes.

\section{Acknowledgements}

This research was financially supported by non-tax revenue (BNPB), Diponegoro University, Semarang, Indonesia under contract no. 1983/UN7.5.8/PP/2020

\section{References}

[1] I. Langmuir,"Oscillations in ionized gases," Proc. of the Nat. Ac. of Sci. of the Unit. States of Am., 14, 8, 627, (1928).

[2] F. F. Chen, Introduction to plasma physics. Springer, New York, (1974).

[3] H. Kadete, P. T. M. Vaessen, and P. C. T. Van der Laan,Enhancement of Heat Transfer by Corona Wind. Eindhoven University of Technology, Eindhoven, (1987).

[4] N. Merbahi, O. Eichwald, D. Dubois, A. Abahazem, andM. Yousfi, "Positive point-toplane corona discharge in air: electrical and optical analysis," In Proc. 28th ICPIG15-20 July 2007, 956-963, (2007).

[5] K. Adamiakand P. Atten,"Simulation of Corona Discharge in Point-Plane Configuration,"J. of Electrost., 61, 2, 85-98, (2004).

[6] A. Y. Wardaya, Z. Muhlisin, A. Hudi, J. E. Suseno, M. Nur, A. W. Kinandana, and J. Windarta,"A study of line-plane configuration in the Corona discharge theory," The Europ. Phys. J. Appl. Phys., 89, 3, 30801, (2020).

[7] E. Moreau, N. Benard, F. Alicalapa, and A. Douyère, "Electrohydrodynamic force produced by a corona discharge between a wire active electrode and several cylinder electrodes-Application to electric propulsion,"J. of Electros., 76, 194-200, (2015).

[8] E. Moreau, P. Audier, andN. Benard, "Ionic wind produced by positive and negative corona 
discharges in air,"J. of Electrost., 93, 85-96, (2018).

[9] H. A. Said, H. Nouri, and Y. Zebboudj,“Effect of air flow on corona discharge in wire-to-plate electrostatic precipitator,"J. of Electrost., 73, 19-25, (2015).

[10] M. M. Ohadi, D. A. Nelson, and S. Zia, "Heat transfer enhancement of laminar and turbulent pipe flow via corona discharge,"International Journal of Heat and Mass Transfer, 34, 4-5, 1175-1187, (1991).

[11] J. Taylor, Introduction to error analysis, the study of uncertainties in physical measurements, second edition. California: University Science Books, (1997). 